\title{
2. Über die Verteilung der Kondensationskerne in staubfreier gesättigter Luft, und über ihre Beobachtungsmethoden; $\left.{ }^{1}\right)$ von Carl Barus.
}

1. Kondensationskerne. Verfasser bemerkt, dab die beschriebenen Untersuchungen sich durchweg auf staubfreie Luft beziehen, d. h. die gewöhnlichen oder Aitkenschen Nuclei sind der Luft durch Filtration entzogen. Es ist Sorge getragen, daB die Luft durchweg gesättigt erhalten bleibt (ausgespannte nasse Tücher $u$. dgl.). Die Prüfung geschieht in einer horizontalen zylindrischen Kondensationskammer $45 \mathrm{~cm}$ lang, über $13 \mathrm{~cm}$ weit) aus Glas mit Metalldeckel. Diese ist durch eine weite Messingröhre $(40 \mathrm{~cm}$ lang, $6 \mathrm{~cm}$ im Durchmesser) mit einer großen eisernen Vakuumkammer $(150 \mathrm{~cm}$ lang, über $30 \mathrm{~cm}$ im Durchmesser) verbunden. In der Verbindungsröhre befindet sich der massive Hahn, welcher eine lichte Weite von mehr als $6 \mathrm{~cm}$ haben mub. Ferner ist derselbe mit Öldichtung gegen die äußere Luft zu versehen, um sich zugleich rasch und leicht drehen zu lassen. Die Fig. 1 zeigt zwei solche Apparate ( $V, F$, Vakuum- bzw. Kondensationskammer) nebeneinander stehend. Die Schläuche dienen zur Druckmessung, zu Filtrier- bzw. Nukleationszwecken usw. Die Luft wird zum Teil im reinen Zustande untersucht, zum Teil unter dem EinfluB der $X$-Strahlen oder der $\beta$ - und $\gamma$-Strahlen des Radiums (schwache Exemplare, $10000 X, 100 \mathrm{mg}$, in einer dünnen Röhre aus Aluminium hermetisch verschlossen). Die Strahlen kommen stets von außen. Nach jeder Kondensation müssen die Wasserkerne (vgl. $₹ 8$ ), wenn sie nicht selbst das Beobachtungsmittel bilden, strengstens entfernt werden.

1) Die vollständige Arbeit, auf welcher sich obiger Auszug bezieht, wird in den Carnegie Publications Washington erscheinen; ferner vgl. man Carnegie Publications No. 62. 1907. 
Es handelt sich also in der vorliegenden Mitteilung wesentlich 1. um die Dampfkerne der staubfreien gesättigten Luft (dieselben sind wahrscheinlich Aggregate aus verhältnismäBig wenigen Wassermolekeln); 2. um die Ionen, welche durch ein künstliches oder natürliches äußeres Strahlenfeld in der Kammer selbst hervorgerufen sind; 3 . um die Wasserkerne, welche in staubfreier Luft durch die Verdampfung von Nebelpartikelchen entstehen. Hier ist dann weiter zu unterscheiden zwischen den Wasserkernen, die aus der Verdampfung der Nebel entstehen, welche bzw. auf Lösungskerne (wie die des Phosphor usw.), oder auf Ionen, oder auf Dampfkerne niedergeschlagen wurden. Bei der Eichung wird man am sichersten mit Lösungskernen operieren.

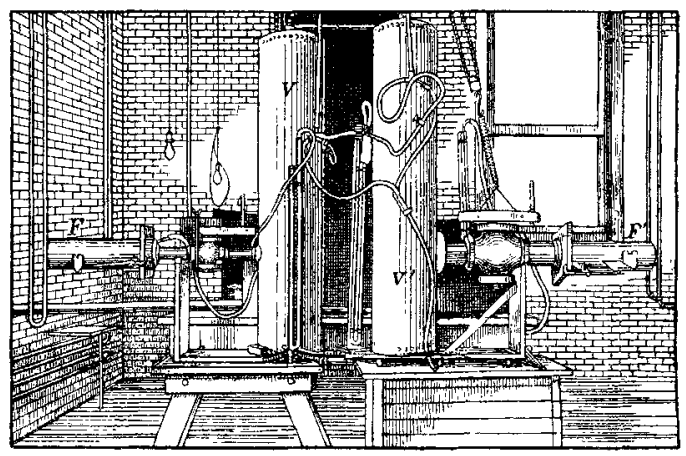

Fig. 1. Nebel- und Vakuumkammern.

2. Beobachtungsmethoden. Die Anzahl der Nuclei bestimmt sich aus dem Winkeldurchmesser der Koronen der nebelartigen Kondensation, bei gegebenem Druck- bzw. Temperaturabfall der gesättigten Luft. Die Eichungsmethode wurde vom Verfasser u. a. in der Boltz mannschen Festschrift (1904. p. 204) beschrieben. Später ist daran vieles verbessert worden. Zieht man von dem künstlich hochnukleierten Medium (am besten eignen sich $P$-Nuclei) bestimmte Mengen durch sukzessive identische Exhaustionen ab, indem man nach der Beobachtung die Nebelkammer jedesmal mit staubfreier Luft nachfüllt, so bilden die aufeinander folgenden Nukleationen (Kernzahlen pro Kubikzentimeter) eine geometrische Progression. Bei den Ver- 
suchen muB man sich an vorbestimmte Zeitintervalle halten, z. B. 5 Sek. zwischen Öffnung des Hahnes und der folgenden Schliebung desselben, sowie der Einleitung der staubfreien Luft, 60 Sek. zwischen aufeinander folgenden Exhaustionen oder dgl. Wegen des geringen Herausfallens der gravitierenden Nebeltropfen ist zu korrigieren. Es verhält sich dann die letzte Nukleation $\left(n_{z}\right)$ zur ersten $n_{1}$, folgendermaßen:

$$
n_{z}=n_{1} y^{z-1} I I\left(1-\frac{S}{s_{z-1}^{2}}\right) \text {. }
$$

Hier ist $y$ die jedesmalige Verdünnung, das Produkt $\Pi$ die Korrektion wegen des Absetzens der Tropfen, wobei $S$ eine Experimentalkonstante und $s_{z-1}$ der Öffnungswinkel der vorhergehenden Korone bedeuten. Wegen anderen Zeitverlusten braucht man bei systematischer Ausführung des Beobachtungsganges nicht zu korrigieren. Letzteren sollte man mit jedem neuen Apparat vorläufig wiederholen.

Um den Winkeldurchmesser der Hauptfarbenringe der Koronen zu bestimmen, bediente sich der Verfasser früher
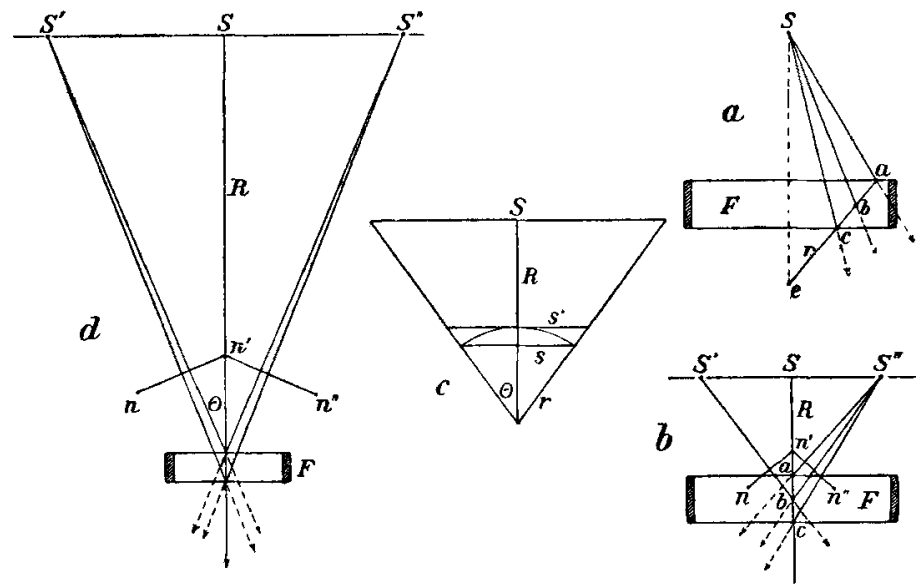

Fig. 2.

eines rasch einstellbaren Goniometers. Der Öffnungswinkel muB stets der Koronenfarbe zugeordnet werden. Es genügt hier eine einzige kleine Lichtquelle (vgl. Fig. 2a). Bei größeren Koronen liegen aber dann die diffraktierenden Nebeltropfen $a, b, c$, welche zugleich Strahlen dem Auge $e$ zusenden, mehr und 
mehr am Ende der Nebelkammer $F$, was zu Refraktionsfehlern Veranlassung gibt. SchlieBlich werden sogar die Strahlen abgeschnitten.

Es würde daher die Methode Fig. $2 \mathrm{~b}$, welche in Fig. 2d skalenmäBig wiedergegeben ist, vorgezogen. Man operiert hier mit zwei identischen, zum Auge bei $c$ symmetrischen Lichtquellen $S^{\prime}, S^{\prime \prime}$, welche sich durch zweckmäBige Mechanismen (Kette und Zahnrad), längs der Schiene $S$, in gleichen Beträgen parallel zur Achse der Nebelkammer zusammenrücken oder voneinander entfernen lassen. Die Beobachtung geschieht in der Weise, daB man die maßgebenden Ringe der beiden Koronen $n n^{\prime}$ und $n^{\prime} n^{\prime \prime}$ bei $n^{\prime}$ zum Kontakt bringt und dann den entsprechenden Abstand $S$, bei festen Radius $R$, mibt. Die Einstellung wird rasch durch eine in der Hand des Beobachters vermittelnde Stange (etwa $250 \mathrm{~cm}$ lang) bewerkstelligt. Ist der Beugungswinkel $\theta$, so gilt $S=2 R \operatorname{tg} \theta$, im Gegensatz zu $S=2 r \sin \theta$ im Falle der Fig. 2a. Die zusammengehörigen GröBen $S, s, s^{\prime}, R, r, \theta$ sind in der Fig. $2 \mathrm{c}$ angedeutet. Die Vorzüge dieser neuen Kontaktmethode bestehen darin, daß die beugenden Nebeltropfen $a, b, c$ in der Äquatorialebene der Nebelkammer $F$ gelegen sind. Man hat es im ganzen mit weniger schiefen Strahlen, daher mit einer geringeren schiefen Refraktion zu tun. Koronen jeder Größe sind beobachtungsfähig, eine sebr wichtige Sachlage, da bei den groBen Koronen die Öffnungswinkel $2 \theta=60^{\circ}$ übersteigen. SchlieBlich kann man beide Augen anwenden und bei $c$ dicht an der Nebelkammer beobachten, was ein bedeutend geschärftesund weniger angestrengtes Sehen ermöglicht.

In praktischer Hinsicht vermeidet man bei Anwendung des Auerstrumpfes die auBerordentlich groBe Farbenkompli. kation, welche die Koronen bei elektrischem Licht zeigen. Weil man die Farbe der Winkelöffnung zuordnen muB, ist das Arbeiten mit Koronen nicht nur mühsam, sondern zahlenmäßig unscharf. Wo alle anderen Beobachtungsmethoden fehlen, wird man es aber gerne anwenden, zumal es bei einem groBen Bereiche von Erscheinungen (z. B. die Molekularstruktur des Dampfes) recht brauchbare Aufschlüsse ergibt.

3. Berechnungsmethode. Beobachten lassen sich nur die Anfangsdrucke $p$ und $p^{\prime}$ der Nebel-bzw. der Vakuumkammer, 
bei der isothermen Temperatur $\tau$ und der Enddruck $p_{3}$, wenn nach der Exhaustion beide Kammern bei derselben Temperatur $\tau$ kommunizieren. Alle anderen Größen, sowohl Drucke wie Temperaturen und Wasserniederschläge, sind aus diesen vorbestimmten Größen in Anbetracht des gegebenen Volumverhältnisses $[\boldsymbol{v} / V]$ zwischen Nebel und Vakuumkammer, der Zustands- und adiabatischen Gleichungen der Gasmasse zu berechnen. So z. B. ist der adiabatische Druck $p_{1}$, wenn sich die gestrichelten Buchstaben auf die Nebelkammer beziehen, und wenn $\pi$ mit Strich oder Index die entsprechenden Dampfspannungen, $c$ und $k$ die beiden spezifischen Wärmen bezeichnen:

$$
\frac{p_{1}-\pi_{1}}{p_{1}{ }^{1-c / k}}=\frac{\left(p_{3}-\pi\right)(1+[v / V])}{p^{\prime(1-c / k)}\left(1-\frac{p_{1}^{\prime}-\pi_{1}}{p_{1}-\pi_{1}}\right)+\left[\frac{v}{V}\right] p^{(1-c / k)}},
$$

wo man rechts anstatt $p_{1}$ den genäherten Wert $p_{3}$ setzen darf. Daher der Vorteil der groben Vakuumkammer. ${ }^{1}$ )

Die Volumausdehnung $v_{1} / v$ der Nebelkammer folgt dann aus $p_{1}$ und $p$; ebenfalls die adiabatisch verringerte Temperatur $\tau_{1}$ aus $\tau$ vor der Kondensation. Ist $\varrho$ die Anfangsdichte (bei $p$ und $\tau$ ), $L$ die latente Wärme der Luft, so berechnet sich der Niederschlag pro Kubikzentimeter analog der von C. T. R. Wilson ${ }^{2}$ ) und J. J. Thomson gebrauchten Näherungsmethode zu

$$
m=\varrho \frac{e}{L} \frac{p_{1}-\pi_{1}}{p-\pi}\left(\frac{p}{p_{1}}\right)^{1-c / k}\left(\bar{\tau}_{1}-\tau_{1}\right),
$$

wo $\overline{\boldsymbol{\tau}}_{1}$ die adiabatische Temperatur der Luft in der Nebelkammer, erhöht durch die Kondensation des Wasserdampfes, bedeutet. SchlieBlich ist $\bar{\tau}_{1}$ durch Hinzuziehen des bekannten Verbältnisses zwischen der Dichte des gesättigten Wasserdampfes und der Temperatur zu bestimmen. Es läßt sich $p_{1}$ oft durch $p_{3}$ ersetzen. Für die Praxis ist es notwendig, eine $m$-Tabelle in bezug auf Druckabfall und Temperatur der Nebelkammer zu berechnen.

AuBer $p, p_{1}, p_{3}, \tau$ sind weitere Beobachtungen an der Nebelkammer bedeutungslos. So ist z. B. in $1 / 4$ Sek. nach der

1) Vgl. C. Barus, Phil. Mag. (6) 13. p. 357. 1907. Gensuer, mit Korrektion eines Fehlers in der adiabatischen Gleichung, im American Physical Review 24. p. 383. 1907.

2) C. T. R. Wilson, Phil. Trans. London 189. p. 298 ff. 1897. 
Evakuation die Temperatur der Nebelkammer auf etwa 60 Proz. des ganzen Intervalles zwischen der adiabatischen $\tau_{1}$ und der isothermen Temperatur $\tau$ gestiegen. Nach 10 Sek. herrscht praktische Temperaturgleichheit.

4. Die grünen Koronen. Teilt man die Koronen in zwei Gruppen ein, diese mit rötlichem inneren Feld oder Hauptringen, jene mit grünlichem Feld, so sind die letzteren zur Vergleichung der laufenden Beobachtungsresultate geeignet. In den sukzessiven identischen Evakuationen der beschriebenen Methode erscheinen sie im Ablauf regelmäBiger Intervalle. Unter den gröberen Koronen zeichnen sich wenigstens drei ${ }^{1}$ ) durch intensiv grüne Färbung aus. Die Durchmesser der zugehörigen Nebelpartikelchen bestimmten sich zu $d_{4}=0,00052 \mathrm{~cm}$, $d_{3}=0,00040 \mathrm{~cm}, d_{2}=0,00023 \mathrm{~cm}$.

Man ist geneigt, diese Zahlen gleichbedeutend mit dem Verhältnis 4, 3,2 zu deuten, was auf einen ersten Zyklus hinweist, dem die grüne Korone mit $d_{1}=0,00013 \mathrm{~cm} \mathrm{zu-}$ geordnet ist. Dem Verfasser ist es niemals gelungen, diese Korone zu verwirklichen; wohl aber wird die grobe rote Korone des ersten Zyklus bisweilen deutlich erhalten, einer Reihe von Partikelgrößen $d_{1}=0,00016 \mathrm{~cm}$ bis $d_{4}=0,00064 \mathrm{~cm}$ entsprechend. Der Winkeldurchmesser $d_{1}$ beträgt hier etwa $60^{\circ}$, woraus sich nicht nur die enorme Gröbe dieser ersten Koronen, wenn das Auge sich in einem Abstand von der Nebelkammer befindet, ergibt, sondern die Notwendigkeit großer Nebelkammern erhellt. Die Existenz dieses ersten Koronenzyklus findet sich im Einklang mit den entsprechenden(roten bis gelben) Axialfarben des Dampfstrahles. Nachher erlischt jede Farbenerscheinung und der Dampf wird klar.

Die Anzahl $n$ der Kondensationskerne, welche einer gegebenen Korone zukommen, hängt wegen $m$ wesentlich von dem plötzlichen Druckabfall $\delta p=p-p_{3}$ ab.

Variiert man also $\delta p$ bedeutend, etwa von $10 \mathrm{~cm}$ in einer ersten Beobachtungsserie bis $20 \mathrm{~cm}$ in einer folgenden, so muB sich aus den $n$-Werten derselben grünen Korone stets derselbe Tropfendurchmesser $d$ berechnen lassen. Es dient dies zur Kontrolle der Eichung.

1) Die kleineren grünen Koronen liegen zu dicht aneinander und werden gewöhnlich nur durch Zufall erbalten. 
Die angegebene GröBenordnung der Nebeltropfen gegebener Koronen hat der Verfasser an anderer Stelle ${ }^{\text {y }}$ durch photographische Aufnahme zu konstatieren geglaubt.

5. C. T. R. Wilsons Ansichten über Zahl und Größe der Nebeltropfen. Hrn. Wilson, dem die schwierige Kondensation des Wasserdampfes auf Ionen, und die schwierigere auf Dampfkerne zuerst gelang, äußert sich in seiner berühmten Abhandlung anders. Während im vorigen Paragraphen dex SchluB gezogen wurde, daB die kleinsten koronenerzengenden Nebeltropfen die Größe $0,0001 \mathrm{~cm}$ übersteigen, veranschlagt Wils on: dak wenn ,all diffraction colors disappear and the fog appears white from all points of view (adiabatic expansion 1,44) ... the diameter of the drops does not exceed one wave length of light or $50 \times 10^{-6} \mathrm{~cm}$ ". Voraussichtlich bezieht sich Wilson hier auf das weiße schleierartige Zentralfeld der großen roten Korone des ersten Zyklus, entsprechend einer Partikelgröße von der Ordnung $d_{1}=0,00016 \mathrm{~cm}$. Die ursprüng. liche Wilson sche Kondensationskammer war nun einer Probierröhre ähnlich ( $2 \mathrm{~cm}$ weit, $5 \mathrm{~cm}$ lang). In einer solchen würdt sich schwerlich die große grüne Korone $d_{3}$ nicht anders als "greenish-white" beurteilen lassen. Verfasser glaubt daher hier eher das Vorhandensein von $10^{6}$ Dampfkernen pro Kubikzentimeter vermuten zu müssen; oder daB dem Wilsonschen letzten "filmy-white" eher einige Millionen als nach seiner Angabe $10^{8}$ Dampfkerne der staubfreien Luft entsprochen haben dürften. Der $\nabla$ ergleich ist deswegen unsicher, wei. Wilson blob zwei grüne Farben angibt. Dasselbe gilt vor. den $W$-Kurven der Figg. 3 und 5, in welchen Verfasser aus den Wilsonschen Farben und Ausdehnungen eine Andeutung der Lage der entsprechenden Kurven versucht hat.

6. Verteilung der Dampfkerne und der Ionen in der staub. freien Luft. In obiger Nebelkammer stellt sich der relative adiabatische Druckabfall $\left(p-p_{1}\right) / p$, welcher bei groBer Vakuum. kammer nahezu gleich $\left(p-p_{3}\right) / p=\delta p$ wird, als bequeme Ver. teilungsvariable dar.

Hier ist $p$ der Anfangsdruck, $p_{3}$ der Enddruck de1 Nebelkammer bei derselben Temperatur und bei kommuni

1) C. Barus, Physik. Zeitschr. 6. p. 718. 1905. 
zierenden Kammern. Es ist also stets auf $p_{\mathbf{3}} \mathrm{zu}$ warten. Die adiabatische Volumausdehnung schreibt sich dann:

$$
\left(v_{1} / v\right)^{k / c}=1 /(1-\delta p / p),
$$

und zwar sind $\left(v_{1} / v-1\right)$ und $\delta p / p$ im allgemeinen ähnliche Größenordnungen.
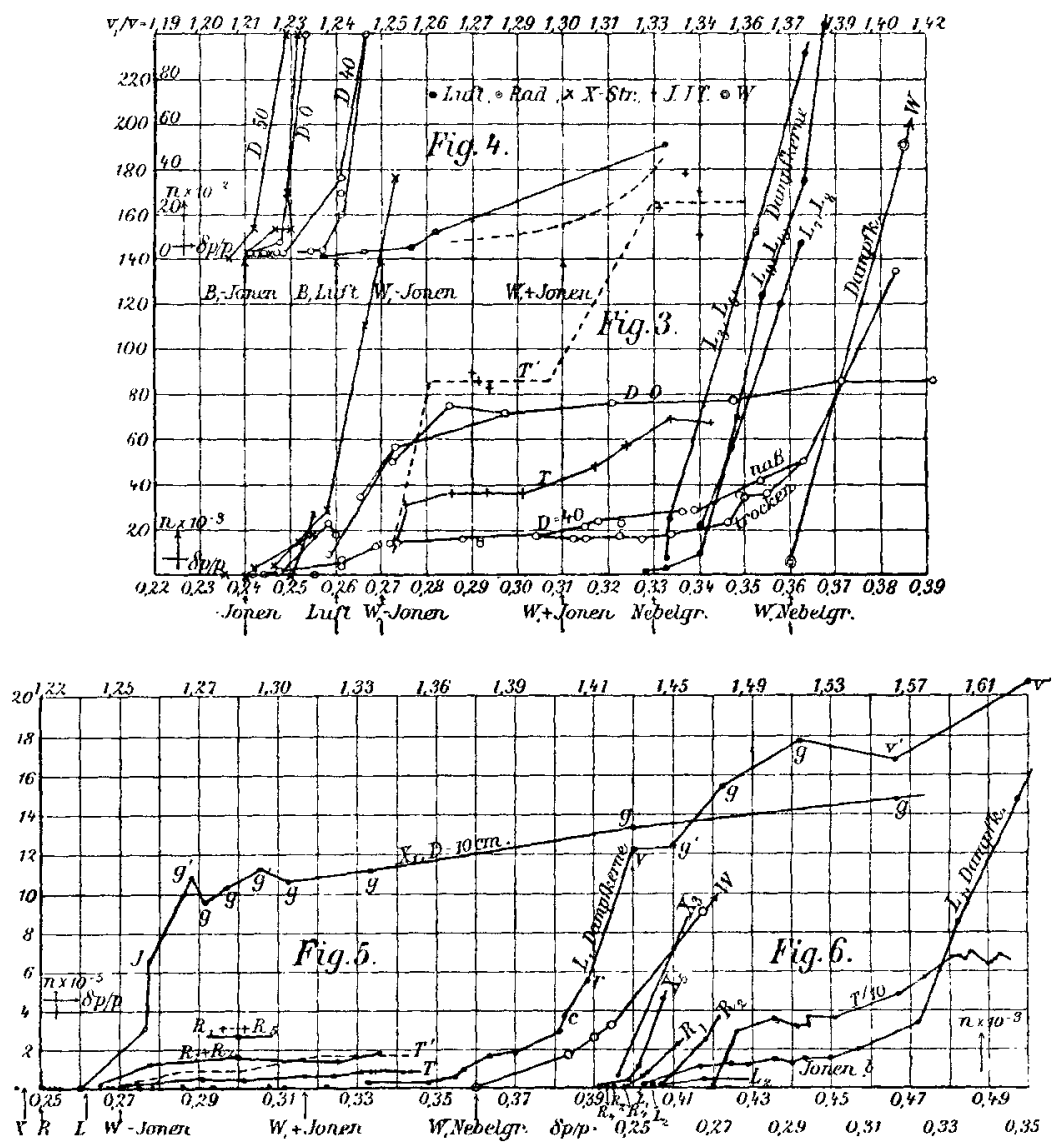

In den Figg. 3, 4, 5 und 6 hat Verfasser eine Übersicht der älteren und neueren Resultate gegeben. Die Ordinate bezeichnet jedesmal die Anzahl $n$ der Kondensationskerne pro Kubikzentimeter in der staubfreien nassen Luft, und zwar in verschiedenen Skalen, je nachdem $n 10^{-2}, n 10^{-3}, n 10^{-5}$. Die Abszisse ergibt (unten) den relativen adiabatischen Druckabfall 
$\delta p / p$, oder auch (oben) die Volumausdehnung $v_{1} / v$. Die Hauptfarben der Koronen sind abgekürzt ( $g$ grün, $y$ gelb, $r$ rot, $c$ tiefrot usw.). $W$ bezieht sich auf Resultate C. T. R. Wilsons ${ }^{1}$, $T$ auf die klassischen Resultate J. J. Thomsons, welche mit Wilson übereinstimmen. $X, R$ beziehen sich auf Einwirkung der $X$-Strahlen, bzw. der $\beta$ - und $\gamma$-Strahlen des Radiums, und zwar aus einer Entfernung $D$ außerhalb der Nebelkammer. $L$ ergibt sich für $D=\infty$ und entspricht also der staubfreien gesättigten Luft. Resultate, welche entweder mit $B$ oder sonst nicht weiter bezeichnet sind, wurden mit verschiedenen Formen des Apparates Fig. 1 erhalten. Verschiedene Beobachtungsserien werden oft durch Indizes $\left(L_{1}, L_{2}\right.$ usw.) bezeichnet.

Bei den Dampfkernen der staubfreien gesättigten Luft unterscheidet man nach Wilson eine Kondensationsgrenze, unterhalb welcher die Kondensation überhaupt aufhört, und eine Nebelgrenze, oberhalb welcher die Koronen rasch anwachsen. Der Verlauf ist aber kontinuierlich. Unter der Abszissenachse sind diese Punkte sowohl für gewöhnliche wie für hochionisierte Luft besonders angegeben.

Die Kurven sind also durchweg Verteilungskurven. Im allgemeinen nimmt die Größenordnung der Kerne mit zunehmendem $\delta p / p$ ab. Bei den Dampfkernen hat man es daher mit der inneren oder Molekularstruktur des Wasserdampfes zu tun and es sind die Resultate in dieser Hinsicht wichtig. Bei gegebenem Temperaturverhältnis usw., finden sich im gesättigten Wasserdampf stets ganz bestimmte Aggregate oder Molekularkomplexe definitiver Größe in ganz bestimmter relativer Anzahl vor. Dieselben werden in gleicher Anzahl sofort erneuert, wie oft auch man sie niederschlagen mag.

Alle Kurven laufen schließlich in einer Asymptote aus, die wahrscheinlich horizontal sein sollte und einer doppelten Interpretation fähig ist. Entweder sind bei der betreffenden Volumausdehnung, bzw. $\delta p / p$, alle Kerne abgefangen, welche der gegebenen Abkühlung ontsprechen (z. B. bei den Kurven der Radiumeinwirkung), oder es läßt die Abkühlfähigkeit, daher die Kondensationsfähigkeit der Nebelkammer nach (z. B. bei

1) Die $W$-Kurven der Figg. 3 und 5 haben nur die Bedeutung eines Versuches, alle vorhandenen Resultate ïbersichtlich darzustellen. Wilson selbst gibt nur Farben und Ausdehnungen an. 
den Kurven für staubfreie Luft). Es ist nicht immer leicht, zwischen diesen Bedingungen zu unterscheiden. Effektiv groBe Kerne verdecken die kleinen mehr und mehr, je nach dem GröBenunterschiede. Nach J. J. Thomson ist die elektrische Ladung einer effektiven Vergrößerung des Kernes äquivalent und es würden sich die ungeladenen Ionen sogar kleiner als die Dampfkerne erweisen.

7. Desgleichen spezielles. In bezug auf die Kerne der gegesättigten staubfreien Luft gibt Figg. 3 und 4 sechs ältere, zu verschiedenen Zeiten gemachte Beobachtungsserien. Die Unterschiede sind wahrscheinlich Temperaturfunktionen, da man einige Prozent Zunahme der Nukleation pro Grad Zuwachs der Temperatur annehmen muB. In den neueren Resultaten, Figg. 5, 6, erlischt die Kondensationsfähigkeit des Apparates oberhalb der grünen Koronen, etwa bei $1,5 \times 10^{6}$ Kernen pro Kubikzentimeter und bei $\delta p / p=0,41$ oder $v_{1} / v=1,45$. Unterhalb dieser Grenze ist die Kurve gut angedeutet. Der Anstieg oder die Nebelgrenze fängt etwa bei $\delta p / p=0,31, v_{1} / v=1,30$ an. Darunter verläuft (vgl. Fig. 6) die vermutete Ionenregion, in welcher etwas über tausend Kerne zugegen sind, sehr allmählich abnehmend bis $\delta p / p=0,262$ oder $v_{1} / v=1,24$. Das ist die rom Apparat (Fig. 1) gegebene Kondensationsgrenze der Dampfkerne. Es liegt dieser Punkt also definitiv unter Wilsons $1,25=v_{1} / v$, welcher bei ihm mit den negativen Ionen zusammenfällt. Setzt man die Luft der Einwirkung des Radiums aus ( $\beta$ - und $\gamma$-Strahlen), so läBt sich die Nukleation über verhältnismäBig niedrige Asymptoten nicht leicht hinaufzwingen. Eine Radiumkapsel $\left.{ }^{1}\right)(10000 X$, $300 \mathrm{mg}$ ), an der Außenwand der Kammer befestigt, gibt im Maximum unter 200000 Ionen, eine solche ${ }^{2}$ ) mit $10000 X$, $700 \mathrm{mg}$, unter 300000 Ionen pro Kubikzentimeter.

Das hängt damit zusammen, daB die Anzahl Ionen, welche pro Sekunde erzeugt werden, schlechtweg (\$ 9) mit dem Quadrat von der Gleichgewichtsnukleation $n$ anwachsen muB, also etwa mit der sechsten Potenz der Koronenöffnung. Es ist also immer die Vergrößerung des Koronendurchmessers durch ein

1) $R_{1}+R_{2}$ in Fig. 5 .

2) $R_{1}+\ldots+R_{\text {б }}$ in Fig. 5 . 
enorm rasches Anwachsen des Erzeugungskoeffizienten bedingt. In dieser Beziehung ist die außerordentlich kräftige Einwirkung der $X$-Strahlen merkwürdig.

Bei den Ionen beginnt aber im Apparat (Fig. 1) die Kondensation bereits wesentlich unter $\delta p / p=0,26$ oder unter $v_{1} / v=1,23$, also deutlich unterhalb des Punktes für staubfreie Luft oder des Wilsonschen Punktes für negative Ionen, welcher letzterem gleichbedeutend ist.

SchlieBlich erniedrigen die $X$-Strahlen die Kondensationsgrenze der negativen Ionen noch weiter, bis auf etwa $\delta p / p=0,247$ oder etwas unter $v_{1} / v=1,223$, deutlich unterhalb des nämlichen Punktes der Einwirkung des schwachen Radiums. Im Vergleich mit den Luftkurven (Dampfkerne) ist der Anstieg bei der starken Ionisation im Feld der $X$-Strahlen ebenfalls steiler, fast plötzlich. Verfasser konnte aber im obigen Apparat nicht über die grünen Koronen hinauskommen, d. h. es lieBen sich etwa $10^{6}$ Ionen bei dem adiabatischen Druckabfall $\delta p / p=0,29$ oder $v_{1} / v=1,27$ abfangen. Darüber hinauszukommen ist entweder deswegen unmöglich, weil der Apparat versagt, oder es sind sämtliche Ionen in der Tat niedergeschlagen. Wahrscheinlich ist letzteres der Fall.

Die Dampfkerne der gesättigten Luft in der Gegenwart der Ionen als Kondensationskerne zu verwenden, gelingt im allgemeinen nicht. Die Ionen beanspruchen den ganzen Wassergehalt der Luft für sich. Nur bei schwächerer Ionisation (vgl. Fig. 3 ; etwa bei $n=20000$, z. B. im Falle des Radiums, $100 \mathrm{mg}, 10000 X$, im Abstand $D=40 \mathrm{~cm}$ ) kommen die Dampfkerne bei erhöhter Ausdehnung, $v_{1} / v$, zur Geltung. Die Kurve verläuft dann auch etwas anders, je nachdem das Glasinnere durch stetiges Umspülen naß oder verhältnismäBig trocken (nicht umspült) erhalten wird. Auch bei etwas stärkerer Ionisation, $n=40,000$ etwa, fängt man noch Ionen und Dampfkerne zugleich auf, aber nur bei sehr starker Exhaustion. Die Kondensation ist dann diffus und koronenlos.

Bemerkenswert ist, daB in Nebelkammern obiger Art, sowohl die Kondensationsgrenzen wie die Verteilungskurven durchweg unter den Werten zu liegen kommen, welche Wilson (und andere, vgl. w. u.) in seinem Kolbenapparat ermittelten. Man vergleiche die $W$-Punkte in Figg. 3 bis 6 mit den übrigen. 
Verfasser findet auBerdem einen deutlich ausgesprochenen Gang der Kondensationspunkte mit der Stärke des Strahlenfeldes, während bei Wilson alles fest sitzt. Beurteilt man die Intensität der Strahlung nach dem Maximum $n_{0}$ der erzeugten Ionen (Asymptote), so liegen folgende Werte vor:

$\begin{array}{llrl}\text { Staubfreie gesättigte Luft } & v_{1} / v=1,24 & n_{0} \times 10^{-3} & =1,5 \\ \beta \text { - und } \gamma \text {-Strahlen . . . } & 1,225 & n_{0} \times 10^{-3}=100 \text { bis } 150 \\ X \text {-Strablen . . . . . } & 1,22 & n_{0} & =10^{6} \text { bis } 2 \times 10^{6}\end{array}$

Nun verhält sich die Stärke des Strahlenfeldes schlechtweg (§9) wie $n_{0}^{2}$, d. h. wenn die Strahlungsintensität von 1 bis $10^{4}$ oder 1 bis $10^{6}$ wächst, so fallen die Kondensationsgrenzen $v_{1} / v=1,24$ um 1,2 bzw. 1,6 Proz. ab.

In den Figg. 3, 5, 6 (in letzterer zehnfach verkleinert) sind die Ionisationswerte, welche J. J. Thomson mit dem Wilsonschen Apparat bei Gelegenheit seiner e-Bestimmung erhielt, unter $T$ und $T^{\prime}$ zur Übersicht eingetragen. Diese beziehen sich selbstverständlich auf die Wilsonschen Kondensationsgrenzen. Die beiden, den negativen sowie den positiven Ionen entsprechenden Stufen im Verhältnis $n_{-}: n_{-}+n_{+}=1: 2$, welche Wilson ${ }^{1}$, Thomson ${ }^{2}$ ), Przibram ${ }^{3}$ ) mit dem Wilson. schen Apparat erkenmen, lassen sich in obiger Kondensationskammer nicht nachbilden. Es ist dies etwas schwer zu erklären, weil sich die Dampfkerne in Gegenwart der mäBigen Ionenzahl sehr wohl abfangen lassen. Es müssen daher sämtliche positive Ionen, auf welche die Kondensation viel leichter stattfindet als auf Dampfkerne, vorher zur Fällung gebracht worden sein.

8. Wasserkerne. Verhalten bei sukzessivem identischen Druck. abfall. Wasserkerne werden besonders bei der Verdampfung von Nebeltropfen erhalten, und zwar am bequemsten bei der Kompression vermittelst der durch das Filter hereintretenden staubfreien Luft. Dieselben unterscheiden sich je nach den Kondensationskernen, auf welchen die Nebeltropfen niedergeschlagen wurden. Unter vielen Unterscheidungsmethoden, welche Verfasser probierte, bewährte sich die oben vermerkte der sukzessiven Partialexhaustionen am besten. Die Kerne

1) C. T. R. Wilson, Phil. Trans. London 189. p. 265-307. 1897.

2) J. J. Thomson, Phil. Mag. (6) 5. p. 346. 1903.

3) H. Przibram, Wiener Ber. 115. Abt. IIa. Januar 1906. 
werden durch die zahlenmäßig aufeinanderfolgenden gleichen Ausdehnungen, mit EinschluB des Gravitierens, schlieBlich zum Verschwinden gebracht.

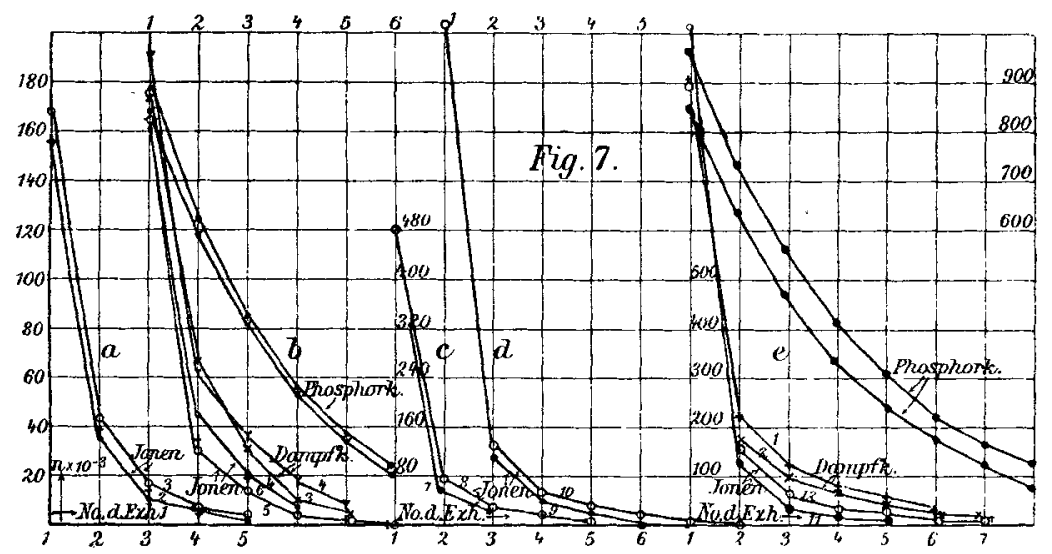

Einige typische Versuchsreihen mögen in Kurven wiedergegeben werden. In Fig. 7, $a$ bis $e$, gibt die Abszisse die laufende Zahl der Partialexhaustion an, die Ordinaten zeigen die Anzahl der Wasserkerne pro Kubikzentimeter bzw. die Anzahl der Nebeltropfen, die sich darauf niederschlagen. Zwischen je zwei Exhaustionen findet in möglichst kurzer Zeit die entsprechende Verdampfung statt. Bei den ersten Exhaustionen wird natürlich auf den Originalkernen präzipitiert, wonach die Kurven benannt sind. Verwendet wurden Lösungskerne (Phosphoremanation), Ionen und Dampfkerne in staubfreier gesättigter Luft. Man überblickt sofort, daB das Verhalten dieser Kerne den sukzessiven Verdampfungen gegenüber ein ganz verschiedenes ist. Die Anfangs- $n$-Werte sind bedeutend variiert.

Knüpft man an obiger Gleichung für die $z^{\text {sche }}$ Nukleation $n_{z}$ in bezug auf die erste $n_{1}$ an, welche sich für Lösungskerne bewährt, so muB man nunmehr einen neuen Koefizienten $(1-x)$ einführen, welcher den relativen Verlust bei den Verdampfungen der Reihe nach anzeigt. Nun ist $x$ für jede Verdampfung verschieden, und zwar mit wachsender Anzahl abnehmend. Daher gilt

$$
n_{z}=n_{1} y^{-1} x x^{\prime} x^{\prime \prime} \ldots \Pi \text {. }
$$


Diese Überlebungskoeffizienten $x, x^{\prime}, x^{\prime \prime} \ldots$ stellt man am besten in bezug auf den Durchmesser $d$ (in Zentimetern) der ver-
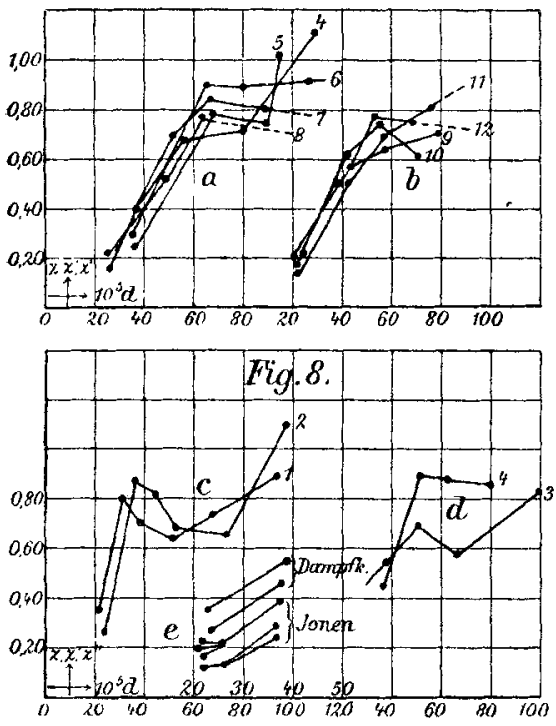
dampfenden Nebeltropfen dar, wie dies in Fig. 8, $a$ bis $e$ ausgeführt ist. In Fig. $8, a, b$ wurden als Originalkerne Ionen angewendet, und zwar in den neun Beobachtungsserien in sehr verschiedener Anzahl (vgl. Fig. 7); in Fig. 9, $c, d$ hingegen befinden sich vier Beobachtungsreihen mit verschiedener Anzahl der Dampfkerne der staubfreien gesättigten Luft. Bei Lösungskernen (Phosphoremanation) ist $x=1$. Fig. 8 , e zeigt das parallele Verhalten im Falle von Dampfkernen und von Ionen in den ersten Exhaustionen.

Man übersieht also, daB bei Lösungskernen, die Dampfdruckerniedrigung durch Lösung (bei fortschreitender Verdampfung) schlieBlich die Dampfdruckerhöhung infolge der Krümmung kompensiert und überkompensiert. Daher ist $x=1$. Bei Wasserkernen, welche auf Ionen und Dampfkernen niedergeschlagen wurden, ist dies im ganzen nur bedingterweise der Fall. Es findet bei jeder Verdampfung ein Verlust, $1-x$, an Wasserkernen statt. Dieser Verlust ist um so größer, je kleiner die Nebeltropfen. $\mathrm{Er}$ ist weitaus am bedeutendsten in der ersten Verdampfung der Nebeltropfen ausgesprochen, wo er $(1-x)$ über 80 Proz. betragen kann. Der Verlust der Verdampfung ist ferner (caet. par.) gröBer bei ursprünglich auf Ionen niedergeschlagenen Wasserkernen als bei solchen, die auf Dampfkernen niedergeschlagen wurden (vgl. Fig. 8,e). Besonders bei letzteren spricht sich die GröBe der Anfangseva. poration aus. Welche Bedeutung dem ferneren Verlauf des Koeffizienten $x$ in Fig. 8, $c, d$ zuzuschreiben ist, läßt Verfasser 
dahingestellt. Wegen der Kleinheit dieser Werte sind mehr als drei, $x, x^{\prime}, x^{\prime \prime}$, deswegen schwer zu erhalten, weil die Koronen rasch verschwinden. $\mathrm{Zu}$ bemerken wäre noch, daB die Ionen, wenn auch klein, in bezug auf die Wasserkerne, dennoch in der Gegenwart der letzten niedergeschlagen werden. Man muB sich also stets befleiBigen, das Strahlenfeld ganz zu beseitigen, ehe man auf Wasserkerne prüft.

J. J. Thomson ${ }^{1}$ ) erklärt die Existenzfähigkeit der Wasserkerne im Falle, wo keine Lösung vorliegt, als Folge des Reinold-Rückerschen Minimums der Oberflächenspannung ${ }^{2}$ ) bei verschwindender Schichtendicke. Wenn sich Nebeltropfen auf den Dampfkernen der staubfreien Luft niederschlagen, so müBte ja ideal reines $W$ asser in jedem Partikelchen vorliegen. Warum aber nur ein kleiner Teil dieser Nebeltropfen durch die übrigbleibenden Wasserkerne repräsentiert wird, wäre noch zu erledigen. Man könnte glauben, daB unterhalb eines gewissen Durchmessers $d$ die Nebeltropfen vollständig verschwinden, oberhalb desselben aber Wasserkerne bilden. Das ist aber nicht der Fall; insbesondere weil ja alle Wassertropfen derselben Korone beisteuern. Ferner wäre der kritische Reinold-Rückersche Durchmesser bedeutend viel kleiner, als den Nebeltropfen zukommt. DaB die Zeitdauer zwischen den Verdampfungen keinen entsprechenden EinfluB hat, hat Verfasser dadurch geprüft, daB in verschiedenen Beobachtungsreihen Intervalle von $1,2 \mathrm{bzw}$. $6 \mathrm{Min}$. eingehalten wurden. Im Verlauf der Zeit zeigen überhaupt die verschiedenen Wasserkerne keine bemerkenswerten Unterschiede. Was die Verschmelzung der Nebeltropfen bei Kollision usw. infolge der Unruhe während der Verdampfung anbetrifft, so müBte dies ja im gleichen Verhältnis ebenfalls bei Phosphornuclei zutreffen.

Es scheint also ein Verhalten eigener Art vorzuliegen, und Verfasser hat ursprünglich die ganze Erscheinung, sowohl bei Lösungskernen als bei Ionen und Dampfkernen (wahrscheinlich irrtümlich), als Lösungsphänomen aufzufassen gesucht.

1) J. J. Thomson, Conduction of Electricity through Gases, Cambridge. chap. VII. p. 152-154. 1903.

2) Mit besonderer Rücksicht auf die langsam sich bewegenden Ionen haben Langevin und Bloch den Gegenstand eingehend verfolgt. 
9. Verlust der Ionen (geladenen Kondensationskernen) in gesättigter Luft im Laufe der Zeit. Da die Verschwindungskoeffizienten der Ionen aus rein elektrischen Messungen bekannt sind, so könnte man daran denken, die Koronen in bezug auf die Kernzahl $n$ auf diese Weise zu eichen. Ein gegebenes Strahlfeld erzeuge $a$ Kerne pro Kubikzentimeter pro Sekunde, während $b n^{2}$ verschwinden. Dann ist:

$$
d n / d t=a-b n^{2} \text {. }
$$

Wird nun das Strahlfeld $t$ Sekunden ror der Exhaustion abgeschnitten $(a=0)$, so gilt:

$$
1 / n=1 / n_{0}+b\left(t-t_{0}\right)
$$

Kennt man nun die relative Nukleation $n / n_{0}$ (man könnte sie z. B. wie in $\& 2$ ermitteln), so ließe sich die absolute Nukleation berechnen, $d . \mathrm{b}$.

$$
n_{0}=\left(n_{0} / n-1\right) / b\left(t-t_{0}\right)
$$

Diese einfache Vermutung des Verfassers stieB aber in den Versuchen auf ungeahnte Schwierigkeiten, die darin fußten, daB der in der Nebelkammer ermittelte Koeffizient $b$ gewöhnlich über dreimal die Größenordnung $b=10^{-6}$ des elektrischen Koeffizienten übertrifft, während er bei abnehmender Kernzahl wohl über das Zehnfache anwachsen kann. Folgendes Beispiel möge herausgegriffen werden:

$\begin{array}{rccc}\text { Zeit } t & \begin{array}{c}\text { Nukleation } \\ n \times 10^{-3}\end{array} & \begin{array}{c}b \times 10^{8} \\ \text { (sukzessiv) }\end{array} & \begin{array}{c}b \times 10^{8} \\ \text { (Gruppen) }\end{array} \\ 0 \text { Sek. } & 83 & 2,9 & 3,8 \\ 5 \quad " & 38 & 2,1 & 4,1 \\ 10 " & 27 & 3,3 & 5,7 \\ 20 " & 14 & 4,2 & 13,4 \\ 30 " & 9 & 3,5 & \\ 60 " & 5 & 15,0 & \\ 120 " & 1 & & \end{array}$

Man könnte nun glauben, daB sich durch die Exhaustion nur ein Teil der vorhandenen Ionen niederschlägt, z. B. der $m^{\text {te }}$ Teil. Dann wäre allerdings:

$$
-d n / d t=m b n^{2},
$$

oder der Koeffizient $b$ wäre vermfacht. Auf diese Art erklärt sich aber nicht, warum $b$ bedeutend zunimmt, wenn $n$ ab- 
nimmt. Die Versuche zeigen ferner, da $B \quad b$ ganz allgemein mit $1 / n$ wächst, sei es, daB die geringe Nukleation durch schwaches Strahlfeld (wenige Kerne erzeugt), oder durch geringe Exbaustion (wenige Kerne abgefangen), oder endlich durch Verluste im Laufe der Zeit (es bleiben nur wenige der ursprünglichen Kerne übrig) erhalten wurde.

Verfasser hat nun zunächst die Annahme, welche ihm bei seinen Versuchen mit Phosphorkernen im Dampfstrahl gute Dienste leistete, herangezogen. Man mache den Ansatz, dab neben dem Verlust durch gegenseitige Koalescenz $b n^{2}$ ein Absorptionsverlust $c n$ besteht. Dann gilt also:

$$
d n / d t=a-b n^{2}-c n,
$$

oder integriert für den Fall $a=0$ :

$$
1 / n=1 / n_{0}+\left(1 / n_{0}+b / c\right)\left(\varepsilon^{c\left(t-t_{0}\right)}-1\right) .
$$

Koronenversuche sind nun zahlenmäBig nicht scharf genug, um zwischen $b$ und $c$ genau zu unterscheiden. Konstantenpaare kompensieren sich. Setzt man aber $b=10^{-6}$, so wird $c=0,036$. Auf diese Weise sind die fünf Beobachtungsreihen in der Fig. 9, $a, b, c$ dargestellt, wo die Beobachtungsresultate

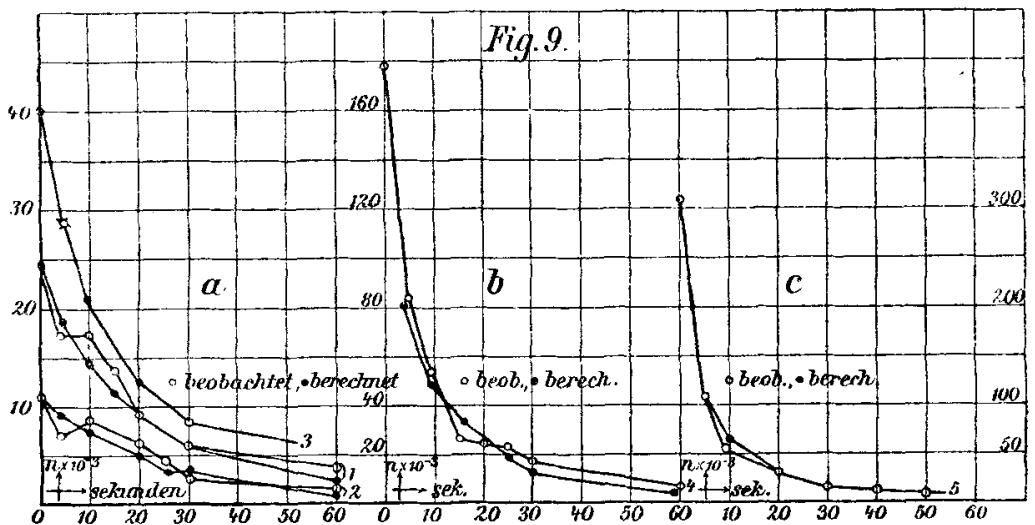

selbst durch kleine Kreise von den berechneten Werten unter. schieden sind. Die Übereinstimmung ist, in Anbetracht der Art der Versuchsbeschränkungen, eine befriedigende.

Man bemerkt, daß der Koeffizient

$$
\frac{d n / d \cdot t}{n^{2}}=\frac{c}{n}+b
$$

Annalen der Physik. IV. Folge. 24. 
nur bei sehr großen $n$ mit $b$ übereinstimmt; bei kleinen $n$ aber über alle Grenzen wachsen kann. Ist z. B. $n=10^{6}$, so ist der Einfluß des $c / n$ kaum merklich; fällt aber die Nukleation auf $n=10^{4}$ herab, so muB $c / n$ bereits bedeutend überwiegen, in derselben Art, wie sich der Koeffizient aus den Beobachtungen selbst faktisch entnehmen läBt.

Man könnte daran denken, daB sich bei der Eichung systematische Fehler eingeschlichen haben. Wäre z. B. $n$ die wahre, $N$ die akzeptierte Nukleation, und $N=A+B n$, wo $A$ und $B \cdot$ Konstanten sind, so ergebe sich zwar

$$
-d N / d t=b^{\prime} N+c^{\prime} N+d^{\prime} \text { für } a=0 .
$$

Nun verschwinden aber $c^{\prime}$ und $d^{\prime}$ wie die erste und zweite Potenz von $A$, während $\operatorname{dem} A$ kein Sinn beizulegen ist.

SchlieBlich wird das Quadrat der Gleichgewichtsnukleation $d n / d t=0$ bei einem gegebenen Strahlenfeld nicht mehr die Intensität $a$ messen. Es ist nun

$$
n=\frac{c}{2 b}-\left(1+\sqrt{1+4 a b / c^{2}}\right) .
$$

Wollte man also die Eichung der Nebelkammer dadurch vermitteln, daß man verschiedene Radiumkapseln, deren Strahlfelder einzeln die Nukleationen $n_{1}, n_{2}, n_{3} \ldots$ erwecken, so ließe sich das Gesamtfeld $n$ nur dann $n=\sqrt{n_{1}^{2}+n_{2}^{2}+n_{3}^{2}+\ldots}$. setzen, wenn $c=0$ wäre. Versuche dieser Art hat Verfasser auch ausgeführt. Die Interpretation wird aber dadurch erschwert, daB die Nukleation in verschiedenen Teilen der großen Nebelkammer verschieden ist, nämlich von dem Abstand $D$ eines Punktes innerhalb der Kammer und dem in der Radiumkapsel abhängt.

Versuche, die Eichung der Koronen so auszuführen, daß man das Thomsonsche $e$ als Konstante einführt und die Ionisation (Kondensator) bzw. die Nukleation (Koronen) zugleich mißt, sind noch nicht erledigt.

Provi,dence, Rhode Island, U.S.A., Brown University. (Eingegangen 14. August 1907.) 